Is impaired classification of subtle facial expressions in children with autism spectrum disorders related to atypical emotion category boundaries?

Whitaker, L. R, Simpson, A., \& Roberson, D. 


\begin{abstract}
Impairments in recognizing subtle facial expressions, in individuals with autism spectrum disorder, may relate to difficulties in constructing prototypes of these expressions. Eighteen children with predominantly intellectual low-functioning ASD (LFA, IQ<80) and two control groups (mental and chronological age matched), were assessed for their ability to classify emotional faces, of high, medium and low intensities, as happy or angry. For anger, the LFA group made more errors for lower intensity expressions than the control groups, classifications did not differ for happiness. This is the first study to find that the LFA group made more across-valence errors than controls. These data are consistent with atypical facial expression processing in ASD being associated with differences in the structure of emotion categories.
\end{abstract}

Key words: facial expression; autism spectrum disorder; intensity; children; categorization; emotion 


\section{Is impaired classification of subtle facial expressions in children with autism spectrum disorders related to atypical emotion category boundaries?}

There is robust evidence that many individuals with autism spectrum disorder (ASD) experience impaired classification of facial emotions. However, this impairment is often subtle. Most research has reported intact classification of high intensity facial expressions in individuals with intellectually high-functioning autism spectrum disorder (HFA); but impaired classification of less intense expressions (e.g., Law Smith et al., 2010; Uono, Sato \& Toichi, 2014). Even then this impairment is usually restricted to negative emotions: happiness is classified well, even when the intensity is low. We examined these difficulties in a group of children with predominantly intellectual low-functioning ASD (LFA - individuals with intellectually LFA are generally accepted in the academic field as an IQ < 80). By examining the processing of varying intensities of emotional expression in an LFA group, we could investigate whether a similar pattern of difficulties is present within this under researched sub-group of ASD.

Theoretical explanations for the impairment of expression recognition in children with ASD draw on the literature concerning how this process unfolds in typically developing (TD) children (Rump et al., 2009; Rutherford \& McIntosh, 2007). One proposal from this literature is that TD children build on their basic emotion prototypes, and hone their representations of what typical expressions looks like (Love, Medin \& Gureckis, 2004), to enable them to identify less intense expressions. In consequence the difficulties children with ASD have, when recognizing less intense expressions, may be underpinned by a failure to develop sufficiently fine-grained prototypes of these expressions (Rump et al., 2009). However previous research has not examined whether impaired recognition of less intense expressions is associated with a deficit in the structure of emotional category boundaries.

TD children initially group emotions by positive and negative valence, confusing emotional labels within the same valence. For example, confusing 'sad' and 'angry' when recognizing angry expressions (two-to-five years of age: Widen \& Russell 2008; two-to-nine years of age: Widen, 2013; Widen \& Russell, 2013) and 'positive surprise' and 'happiness' when recognizing happy expressions (three years to adulthood: Montirosso, et al., 2010; Roberson et al., 2012- two-to-five years of age: Widen \& Russell 2008). Rarely do TD children confuse negative with positive expressions. Infants as young as 6-months-old can associate the emotion label 'happy' to facial expressions, and by three years can associate it with situational information (i.e., they associate 
the causes and consequences of an emotion - de Haan \& Nelson, 1998; Widen \& Russell, 2003); although even in older childhood happiness is still associated with other positive emotions when expressed at lower intensities (Montirosso, et al., 2010; Roberson et al., 2012). In contrast, children only begin to associate an angry face with the emotion label 'angry' at around three years of age (for a review see Widen, 2013; Widen \& Russell, 2013). Through maturation children add to their emotion category boundaries using additional emotion labels (e.g., disgust), associating a strong facial expression and situational information, to their prototype (Markham \& Adams, 1992; Vicari, Reilly, Pasqualetti, Vizzotto, \& Caltagirone, 2000; Widen \& Russell, 2010). Thus the boundaries of these categories are refined and narrowed, shifting from valence-based categories (i.e., positive versus negative) to discrete-emotional categories (e.g., angry versus sad, Widen \& Russell, 2013).

The structure of emotion category boundaries in ASD is under-researched. The majority of previous research (see Harms, Martin, \& Wallace, 2010 and Uljarevic \& Hamilton, 2012 for reviews) has concentrated on recognition of facial expressions, failing to examine whether individuals with ASD confuse negative and positive expressions or demonstrate more subtle within-valence confusion. Two studies (Eack et al., 2014; Kennedy \& Adolphs, 2012) have found that adults with ASD make within-valence confusions with negative emotions. However, across-valence confusions could not be effectively analyzed in these studies, because insufficient positive emotions were included.

Here we examined whether children with predominantly intellectual LFA demonstrate predominantly within-valence confusions suggesting that they have secure valence-based boundaries similar to those of TD children. We presented the same number of positive and negative emotions so that across-valence confusions could also be detected. Building on previous research this study examined emotional expression of different intensities using an established developmental paradigm (Roberson et al., 2012). We tested anger and happiness because TD children map these facial expressions to the appropriate emotion labels (Widen \& Russell, 2008; 2010; Widen et al., 2015). Sadness and positive-surprise were used as distractor emotions. Sadness was chosen because other negative emotions are often confused (disgust: Widen et al., 2015; fear and surprise: Castelli, 2005). If the ASD group demonstrate across-valence confusion then they would classify sadness and anger as happiness, as well as positive-surprise and happiness as anger.

Overall, we had two aims. First to investigate whether the pattern of impaired classification of less intense expressions is present in individuals with predominantly intellectually LFA. We predicted that children with predominantly intellectually LFA would have greater difficulty classifying less intense facial expressions 
than two control groups (a mental age control group matched on verbal and non-verbal ability, and a chronological age control group). The second aim investigated whether impaired expression processing is underpinned by the way emotion category boundaries are structured in ASD. Would individuals with predominantly intellectually LFA make more across-valence inclusions (e.g. classifying happy faces as angry) than the control groups? Two control groups were tested, one matched on mental age (MA) and a second on chronological age (CA). Comparing ASD performance to these two control groups enabled us to determine whether differences in the way emotion category boundaries are structured in ASD is due to intellectual ability or ASD traits. If the way emotion category boundaries are structured in ASD is due to ASD traits, and not intellectual ability, performance should differ once intellectual ability (MA control group) and CA (CA control group) are taken into account. Thus, the ASD group's performance should differ to that of both the MA and CA control group.

\section{Method}

\section{Participants}

Eighteen children diagnosed with ASD, aged 7 - 15 years (5 females) and two groups of 18 control children were tested (see Table 1). All participants in the ASD group had a confirmed clinical diagnosis. In line with the uneven ASD cognitive profile (Joseph, Tager-Flusberg \& Lord, 2002; Tager-Flusberg, \& Joseph, 2003) performance in the non-verbal ability measure was superior to that of the verbal ability measure (see Table 1). Fourteen of the ASD group were assessed, scoring above the ASD threshold, using the Autism Diagnostic Observation Schedule - Generic (Lord et al., 2000). Three participants in the ASD group had a VIQ of above 80. In the mental age (MA) control group aged 6 - 15 years (6 females), children were matched on verbal ability (British Picture Vocabulary scale [BPVS] $(p>.5)$; Dunn, Whetton \& Pintillie, 1997), non-verbal ability (the Raven's Coloured Progressive Matrices [Raven's CPM]; Raven, Court \& Raven, 1992) ( $p>$.8), and chronological age (CA) ( $p>.6)$. Nine participants from the MA matched control group had a statement of special educational needs (aged 10 - 15 years old), the remaining half did not have a statement of special educational needs (aged 6 - 9 years old). It was not possible to obtain consent from parents to view control participants' statements of special educational need. However, schools confirmed that no participants from the MA (or CA) control group had a clinical diagnosis or a suspected diagnosis of ASD. In the CA control group children aged 10 - 15 years (6 females) were matched on CA $(p>.8)$. All participants from the CA matched control group did 
not have a statement of special educational needs. Ethical approval was obtained from the University of Essex Ethics Committee and informed consent was obtained from the parents of all participants.

\section{Materials}

The face stimuli consisted of colored photographs of facial expressions of four emotions: anger, sadness, positive surprise (models directed to express positive surprise) and happiness, taken from a subset of those generated by Montagne et al. (2006). For each emotion three levels of intensity were presented (100\%, high intensity; $80 \%$, medium intensity and 60\%, low intensity). $60 \%$ and $80 \%$ intensity emotions were developed using a morphing algorithm in which $100 \%$ expressions were morphed with a neutral expression (see Law Smith et al., 2010; Roberson et al., 2012 for evidence of validation of this face set). Luminance, brightness, contrast and skin tone were equated. Anger and happiness were selected as the target emotions to examine classification of one position and one negative emotion. Sadness and positive surprise were selected as the distractor emotions because one was negative and one was positive, making them balanced controls. Two sets of 12 stimuli (4 emotions at 3 levels of intensity) were created: one using a male face and one using a female face. Each face stimulus measured 10 x $15 \mathrm{~cm}$ and was printed on a black background (see Figure 1). Following Roberson et al., (2012) two painted cardboard 3D houses (approx. 22 x 23 x $15 \mathrm{~cm}$ ) were created with a gap in the roof for the stimuli to be posted through.

[Figure 1 here]

\section{Procedure}

Practice trials and test trials followed the same procedure as that of Roberson et al., (2012). Participation in the test phase was conditional on successful completion of the practice trials. For the test phase two cardboard houses were placed on the desk in front of the participant. Happy expression condition: participants were told, "Now we will play a new game. In these houses there are parties for people who are feeling different things. The party in this house is for people who are feeling happy." The second house was then pointed to and the experimenter said, "The party in this house is for everyone else who is not happy". Participants were presented randomly with the 12 face stimuli individually then asked, "Is this person happy? Which house party should s/he be in?" The participant then placed the stimulus in their chosen house. The same procedure was followed for the other target emotion of anger. Order of emotion and house was randomized across participants. The cardboard houses and posting technique suited the low verbal ability of the ASD group and encouraged 
engagement with the task (see Roberson et al., 2012). Half the participants from each group saw the female face set and the remaining half saw the male face set.

Results

\section{Analysis}

For each emotion three of the 12 faces depicted the target emotion at either low (60\%), medium (80\%), or high (100\%) intensity. A response was judged correct if the child 'posted' one of the three target images into the correct house, or correctly excluded one of the nine faces displaying different expressions. Correct inclusions of targets were triple weighted compared to correctly excluding non-targets, since a target emotion (of a specific intensity) could only be included once, but three distractors could be excluded. The triple weighting of correct inclusions avoids the possibility that a participant could exclude everything, but still obtain a relatively high score (i.e., 67\%). This resulted in a maximum total correct score of 6 per intensity (3 for a correct inclusion of a target emotion plus 1 for every correct exclusion of distractors). The results remained unchanged once proportion of correct was calculated without triple weighting correct inclusions of the target emotion. The data were converted to a proportion correct score (0-1).

\section{Effect of intensity on facial expression classification}

Happy and angry data were analyzed separately - angry first (Figure 2). Angry versus not angry classifications were analyzed in a 3 (Group: ASD, MA matched controls, CA matched controls) x 3 (intensity: high, medium and low) mixed design ANOVA, with repeated measures over the second factor. This revealed a significant main effect of group $F(2,51)=6.88, p=.002$, partial $\eta 2=.21$, no significant effect of intensity [ $p>$ .7], but a significant interaction between group and intensity, $F(2,51)=3.18, p=.02$, partial $\eta 2=.11$. When classifying faces as angry vs. not angry, the ASD group made significantly fewer correct classifications than the control groups. Three post-hoc Fischer's Least Significance Difference tests were used to examine the interaction. These revealed that the ASD group was significantly less likely to classify low intensity and medium intensity angry facial expressions as angry than were the CA (low intensity: $p=.028$, medium intensity: $p=.001$ ) and MA control groups (low intensity: $p=.007$, medium intensity: $p<.001$ ). This pattern did not reach significance for high intensity (CA controls: $p>.5$, MA controls: $p>.3$ ). In contrast there were no significant effects in the happy classification analysis (Figure 3).

[Figure 2 and 3 here] 
To investigate sensitivity to emotion category boundaries, the mean number of across-valence inclusions was compared between the ASD and control groups at the three intensities. The proportion of across-valence inclusions was calculated: happy and surprise classified as angry, and sad and angry classified as happy (Table 2).

For angry versus not angry classifications a mixed design ANOVA, where intensity was the withinsubjects factor and group was the between subjects factor, was calculated. The main effect of intensity was not significant $(p>.1)$. The main effect of group was significant $F(2,51)=3.275, p=.046$, partial $\eta 2=.11$. There was also a significant interaction between group and intensity $F(2,51)=3.290, p=.014$, partial $\eta 2=.03$. In three follow-up one-way ANOVAs, examining each intensity separately, the ASD group was significantly more likely to make across-valence inclusions when classify medium intensity facial expressions as angry than controls $F(2,51)=9.00, p=.0001$. This pattern did not reach significance for high $(\mathrm{p}>.3)$ or low intensities (p>.5). Fisher's Least Significant Difference post hoc tests revealed both the MA matched $(p=.002)$ and CA matched $(p=.002)$ control groups were less likely to make across-valence inclusions than the ASD group for medium intensity facial expressions, control groups' performance did not differ ( $p>9$ ).

For happy faces a mixed design ANOVA, where intensity was the within-subjects factor and group was the between subjects factor, was also calculated. The main effect of intensity was significant, $F(1,51)=3.17, p$ $=.046$, partial $\eta 2=.06$. The main effect of group was significant $F(2,51)=4.724, p=.013$, partial $\eta 2=.16$. Group did not interact significantly with intensity ( $p>.2)$. Post hoc comparisons revealed the ASD group was significantly more likely to make across-valence inclusions when classifying happy facial expressions (intensity collapsed) than CA matched controls $(p=.019)$. This pattern broached significance for MA matched controls $(p=.058)$.

\section{Discussion}

First this study assessed the impact of expression intensity on emotion classification in a group of children with predominantly intellectually LFA. In line with previous research (e.g., Law Smith et al., 2010) these children were able to classify happy facial expressions at varying intensities, but were specifically impaired when classifying less intense angry facial expressions relative to the control groups. A finding present when compared to control participants matched on either MA or CA, suggesting that any differences in emotion category boundaries in ASD may be related to ASD traits as opposed to IQ. Second we examined whether impaired expression processing in ASD is underpinned by the way emotion category boundaries are structured, 
thus we analyzed the proportion of across-valence inclusions. This is the first study to find that children with predominantly intellectually LFA made more across-valence inclusions when classifying both angry and happy facial expressions, compared to the control groups (e.g., classifying a happy face as angry and classifying a angry face as happy). Our findings suggest that children with ASD have difficulties processing facial expressions, in that they associate different- as well as same-valence expressions with the emotion label. The persistence of across-valence inclusions in children with ASD suggests that their difficulties are more profound than mere deficits in labeling emotions.

Across-valence inclusions when the target emotion was anger

For the control groups, as in Roberson et al. (2012; Russell \& Widen, 2003), participants made almost exclusively within-valence inclusions when classifying angry expressions, with only eight out of 36 control participants making any across-valence inclusions (three from the MA control group, five from the CA control group). In contrast children with ASD made more across-valence inclusions than controls, with up to half of participants from the ASD group making these inclusions. Indeed the ASD group used more across-valence labels than the control groups at medium intensity facial expressions, which was the level of intensity they found the most challenging. This suggests children with ASD form weaker valence category boundaries than TD children, and that their mental representation for anger is activated by positive-valence facial expressions (happiness, surprise).

Across-valence inclusions when the target emotion was happy

Children with predominantly intellectually LFA showed classification of happy facial expressions that was intact compared to controls. An unsurprising finding in light of the early age happiness is recognized, and ease with which happiness is classified (de Haan \& Nelson, 1998; Ewing, Karmiloff-Smith, Farran \& Smith, 2015). Nevertheless these children with ASD did make more across-valence inclusions (sad and angry) than CA matched controls (a finding that also broached significance for MA matched controls). These findings suggest that while not impaired when classifying happiness, our ASD group did have atypical happiness prototypes. Mental representations for happiness may be activated by across-valence facial expressions in ASD. As with angry expressions, the higher rate of across-valence inclusions with happy expressions suggests that children with ASD have weaker emotion category boundaries.

Overall, this pattern of performance speaks to Rump et al.'s (2009) proposal that children with ASD develop atypical mental representations of facial expressions. Although this particular developmental trajectory 
is unexplored in ASD, the current data suggest that expression classification impairments in children with ASD go beyond labeling difficulties. Future research should consider examining the pattern of across-valence inclusions that children with intellectually HFA and children with intellectually LFA make, to provide an overall understanding of the structure of emotion category boundaries across the autistic spectrum.

\section{Limitations}

To fully determine whether making across-valence inclusions of non-target emotions was dependent on IQ or ASD traits, a MA matched control group who consisted of children with matched and defined special educational needs could be collected. We were not able to obtain MA control participants' statements of special educational need. Furthermore, nine participants from the MA matched control group did not have a statement of special educational need (despite their relatively low IQ). For this reason it was not possible to match the ASD group to a group of controls who all had a statement of special educational needs, or to determine the etiology of language impairments in the control group.

The possible role of language in emotion prototype formation also needs further investigation. This could be achieved in future research by collecting data from a MA matched control group who consisted of children with specific language impairment. In addition, to examine whether making across-valence inclusions of non-target emotions occurs across the autistic spectrum, and to control for the effects of IQ more rigorously, future research could work with children with intellectually LFA and HFA, as well and including a larger group of control participants. Examining a larger group of children with ASD (with a range of language abilities) would also enable the exploration of how language impairment and language acquisition (Stagg, Davies \& Heaton, 2013) relate to emotion prototype formation.

\section{Implications}

Our data suggest that children with predominantly intellectually LFA have difficulty classifying angry and happy expressions of emotion. Greater inclusions of across-valence emotions with angry and happy expressions suggests children with ASD may follow a different pattern of emotion prototype formation than TD children. Difficulties in facial expression classification may relate to both a difficulty mobilizing the emotion label and atypical categorization of emotion concepts. This atypical categorization of emotions may impact on later social interaction causing difficulties developing an understanding of how to integrate multiple emotional cues (facial, situational, auditory) and less intense expressions. 
Table 1: Means (standard deviation in brackets) of chronological age, verbal ability and non-verbal ability of all participants

\begin{tabular}{llll}
\hline & Age & BPVS & Raven's CPM \\
& (years; months) & Standard score & Raw score \\
\hline CA matched control group $(\mathrm{n}=18)$ & $10 ; 7(2.23)$ & $97.6(10.63)$ & $26.7(2.81)$ \\
\hline MA matched control group $(\mathrm{n}=18)$ & $10 ; 5(2.26)$ & $69.1(25.60)$ & $24.8(3.17)$ \\
ASD group (n=18) & & & $24.5(4.51)$ \\
\hline
\end{tabular}

BPVS = British Picture Vocabulary Scale; Ravens CPM = Raven’s Coloured Progressive Matrices 
Table 2 The proportion of across-valence inclusions at each level of intensity $(100 \%, 80 \%$ and $60 \%)$ for angry and happy classifications.

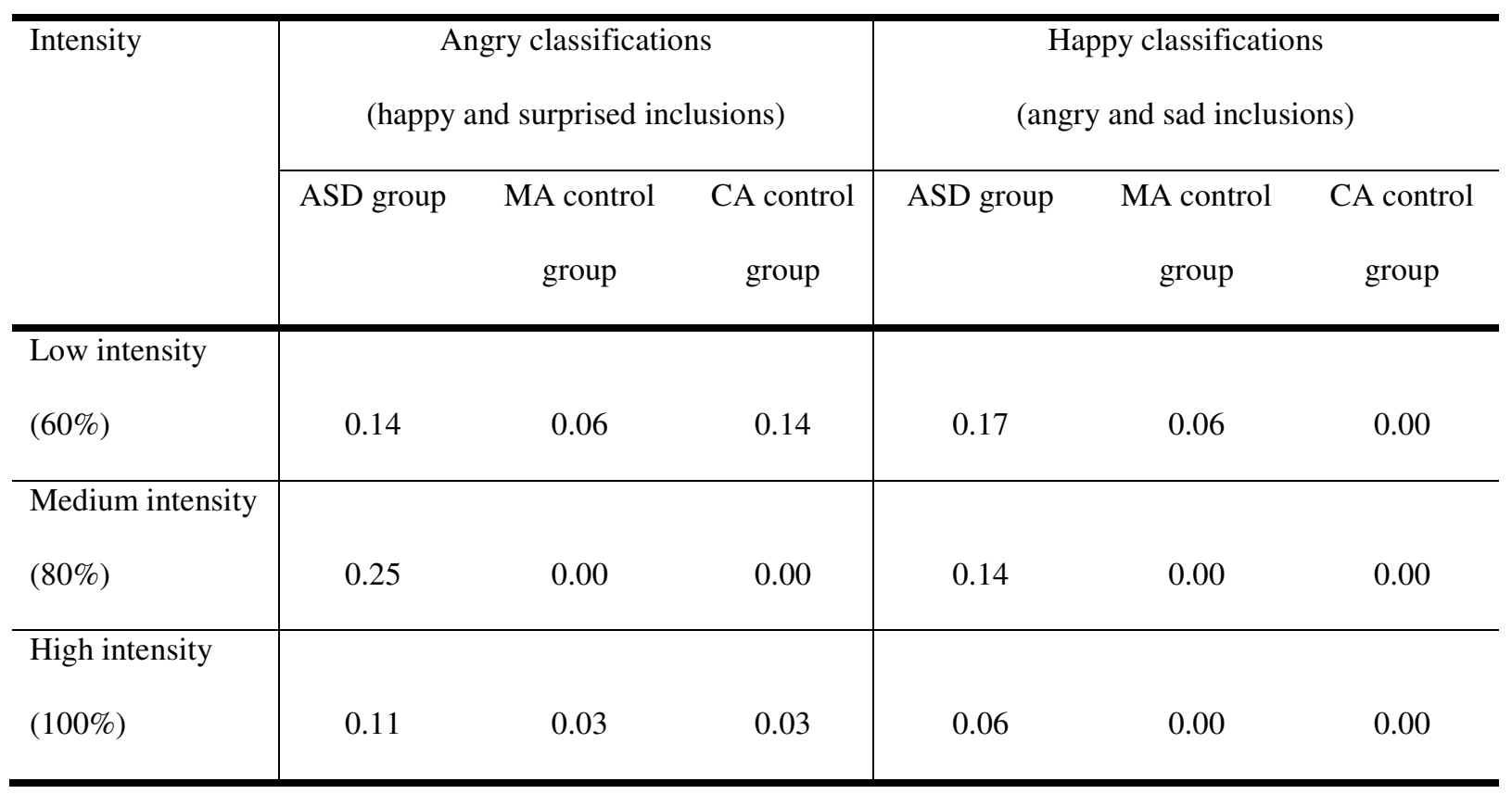


Figures caption sheet

Figure 1 Emotion classification task: The female face expressing anger at 100\%, $80 \%$ and $60 \%$ intensity.

Figure 2 Mean proportion of correct classifications of anger at high, medium and low intensities for the ASD and control groups

Figure 3 Mean proportion of correct classifications of happiness at high, medium and low intensities for the ASD and control groups 
Figure 1

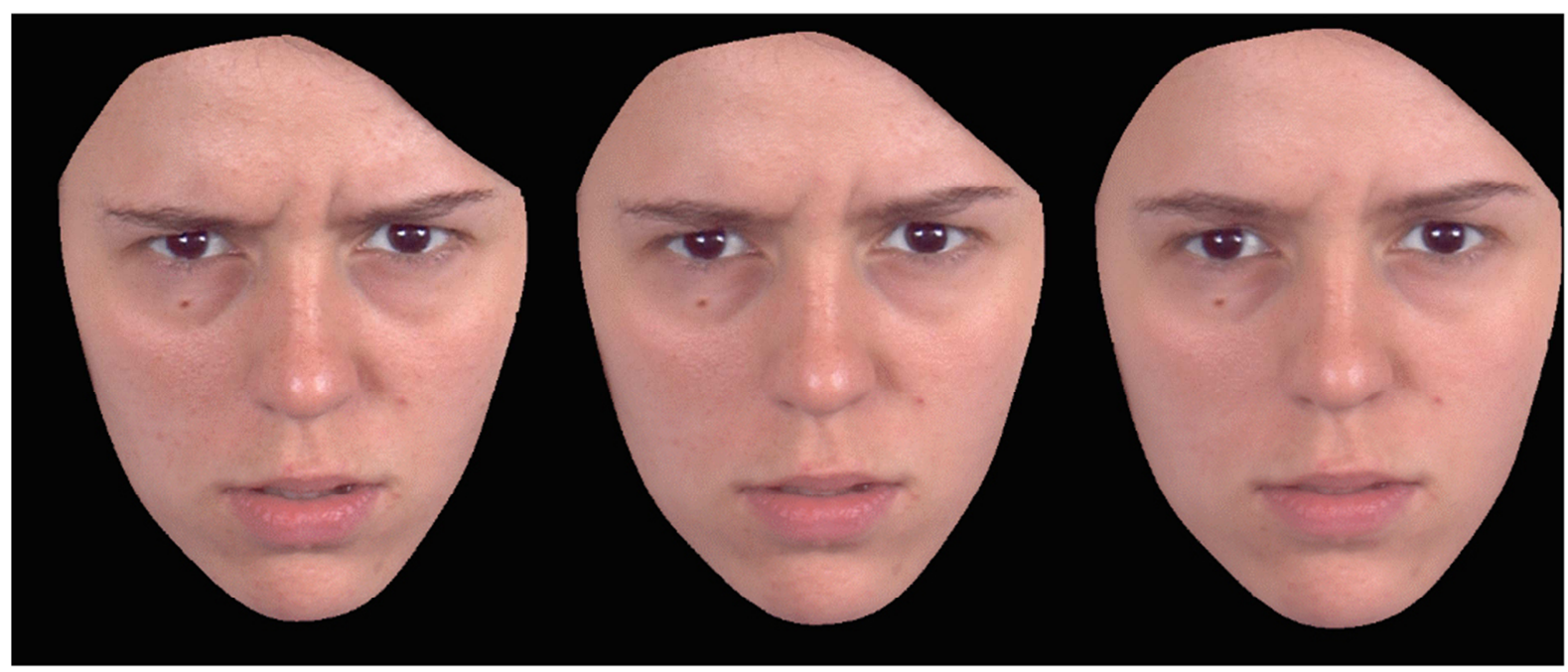


Figure 2

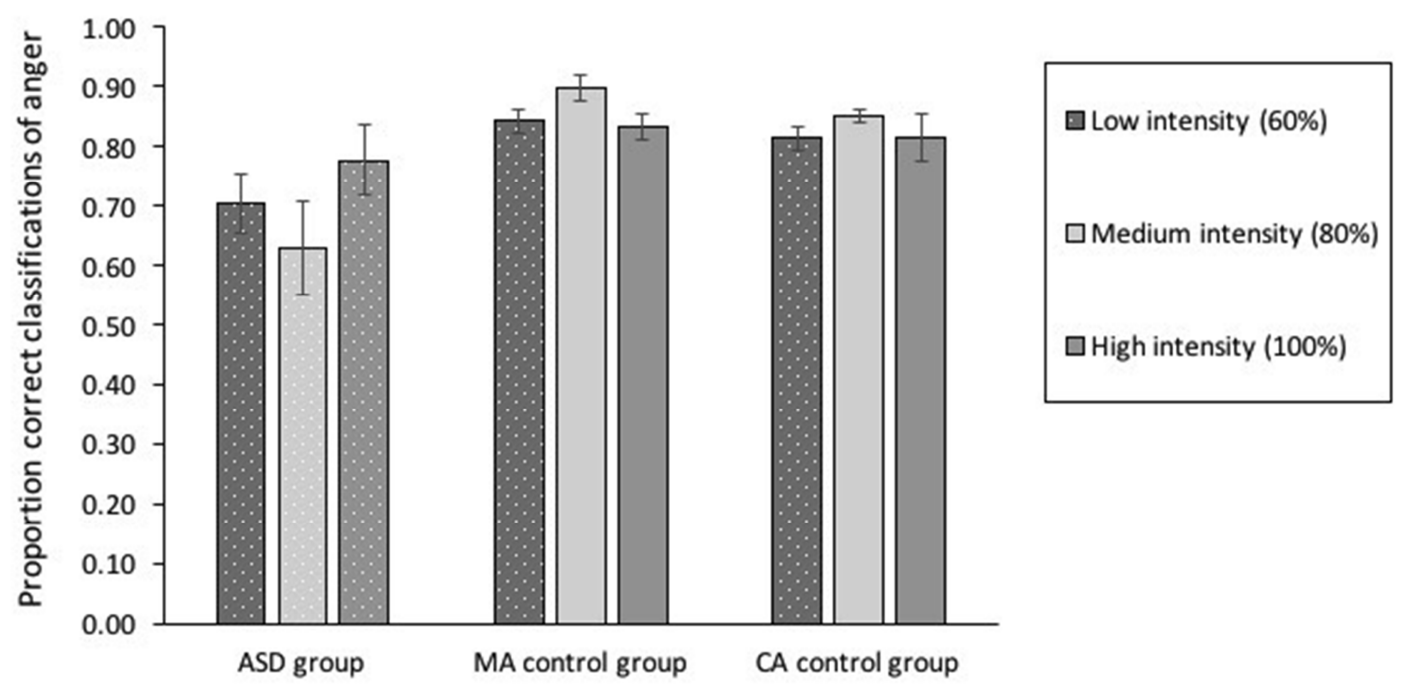


Figure 3

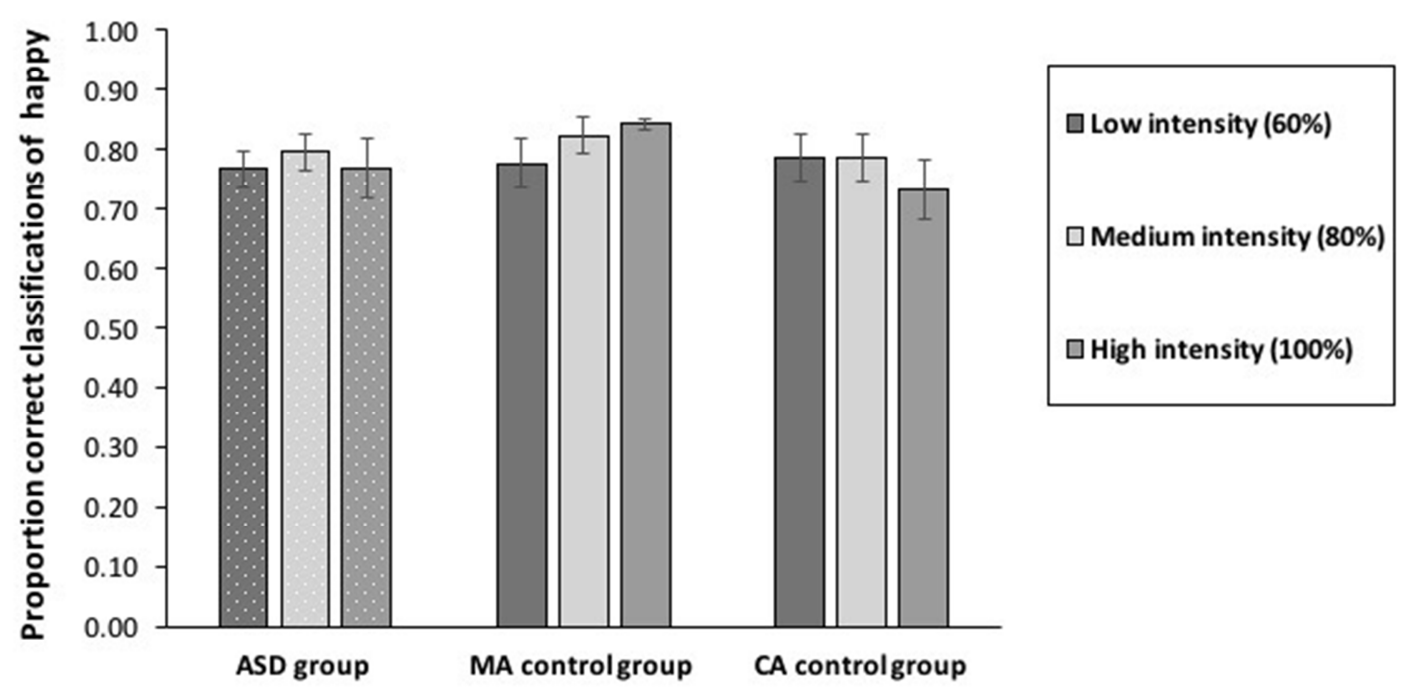




\section{Conflict of interest}

We confirm that none of the above named authors have a conflict of interest to declare. 
Acknowledgments

A University of Essex Studentship to the first author supported this study. 


\section{References}

Castelli, F. (2005). Understanding emotions from standardized facial expressions in autism and normal development. Autism, 9, 428_449.

de Haan, M., \& Nelson, C. A. (1998). Discrimination and categorization of facial expressions of emotion during infancy. Perceptual development: Visual auditory, and speech perception in infancy, 247-286.

Dunn, L. M., Whetton, C., \& Pintillie, D. (1997). British picture vocabulary scale. Windsor: NFER-Nelson.

Eack, S. M., Mazefsky, C. A., \& Minshew, N. J. (2014). Misinterpretation of facial expressions of emotion in verbal adults with autism spectrum disorder. Autism, 1362361314520755.

Kennedy, D. P., \& Adolphs, R. (2012). Perception of emotions from facial expressions in high-functioning adults with autism. Neuropsychologia, 50(14), 3313-3319.

Ewing, L., Karmiloff-Smith, A., Farran, E., \& Smith, M. (2015). Capturing developmental shifts in facial identity and expression processing strategies. Journal of vision, 15(12), 1188-1188.

Joseph, R. M., Tager-Flusberg, H., \& Lord, C. (2002). Cognitive profiles and social-communicative functioning in children with autism spectrum disorder. Journal of Child Psychology and Psychiatry, 43(6), 807821.

Law Smith, M. J., Montagne, B., Perrett, D. I., Gill, M., \& Gallagher, L. (2010). Detecting subtle facial emotion recognition deficits in high-functioning Autism using dynamic stimuli of varying intensities. Neuropsychologia, 48, 2777-2781.

Lord, C., Risi, S., Lambrecht, L., Cook, E. H., Jr., Leventhal, B. L., DiLavore, P. C., . . Rutter, M. (2000). The autism diagnostic observation schedule-generic: a standard measure of social and communication deficits associated with the spectrum of autism. J Autism Dev Disord, 30, 205-223.

Love, B. C., Medin, D.L., \& Gureckis, T. M. (2004). SUSTAIN: A network model of human category learning. Psychological Review, 111, 309-332.

Markham, R., \& Adams, K. (1992). The effect of type of task on children's identification of facial expressions. Journal of Nonverbal Behavior, 16(1), 21-39.

Montagne, B., Schutters, S., Westenberg, H. G., van Honk, J., Kessels, R. P., \& de Haan, E. H. (2006). Reduced sensitivity in the recognition of anger and disgust in social anxiety disorder. Cogn Neuropsychiatry, 11, 389-401. 
Montirosso, R., Peverelli, M., Frigerio, E., Crespi, M., \& Borgatti, R. (2010). The Development of Dynamic Facial Expression Recognition at Different Intensities in 4-to 18-Year-Olds. Social Development, 19(1), 71-92.

Raven, J. C., Court, J. H., \& Raven, J. (1992). Standard progressive matrices. OUP: Oxford Psychologists Press.

Roberson, D., Kikutani, M., Doge, P., Whitaker, L., \& Majid, A. (2012). Shades of emotion: what the addition of sunglasses or masks to faces reveals about the development of facial expression processing. Cognition, 125, 195-206.

Rump, K. M., Giovannelli, J. L., Minshew, N. J., \& Strauss, M. S. (2009). The development of emotion recognition in individuals with autism. Child development, 80, 1434-1447.

Russell, J. A. (1994). Is there universal recognition of emotion from facial expressions? A review of the cross-cultural studies. Psychological bulletin, 115(1), 102.

Rutherford, M. D., \& McIntosh, D. I. (2007). Rules versus prototype matching: Strategies of perception of emotional facial expressions in the autism spectrum. Journal of Autism and Developmental Disorders, $37,187-196$.

Stagg, S. D., Davis, R., \& Heaton, P. (2013). Associations between language development and skin conductance responses to faces and eye gaze in children with autism spectrum disorder. Journal of autism and developmental disorders, 43(10), 2303-2311.

Tager-Flusberg, H., \& Joseph, R. M. (2003). Identifying neurocognitive phenotypes in autism. Philosophical Transactions of the Royal Society of London B: Biological Sciences, 358(1430), 303-314.

Uljarevic, M., \& Hamilton, A. (2012). Recognition of Emotions in Autism: A Formal Meta-Analysis. $J$ Autism Dev Disord.

Uono, S., Sato, W., \& Toichi, M. (2014). Reduced representational momentum for subtle dynamic facial expressions in individuals with autism spectrum disorders. Research in Autism Spectrum Disorders, 8(9), 1090-1099.

Vicari, S., Reilly, J. S., Pasqualetti, P., Vizzotto, A., \& Caltagirone, C. (2000). Recognition of facial expressions of emotions in school-age children: the intersection of perceptual and semantic categories. Acta Paediatrica, 89(7), 836-845. 
Widen, S. C. (2013). Children's interpretation of facial expressions: The long Path from valence-based to specific discrete categories. Emotion Review, 5(1), 72-77.

Widen, S. C., Pochedly, J. T., \& Russell, J. A. (2015). The development of emotion concepts: A story superiority effect in older children and adolescents. Journal of experimental child psychology, 131, 186-192.

Widen, S. C., \& Russell, J. A. (2003). A closer look at preschoolers' freely produced labels for facial expressions. Developmental Psychology, 39(1), 114-128. doi: 10.1037/0012-1649.39.1.114

Widen, S. C., \& Russell, J. A. (2008). Children acquire emotion categories gradually. Cognitive Development, 23, 291-312.

Widen, S. C., \& Russell, J. A. (2010). Differentiation in preschooler's categories of emotion. Emotion, 10, 651.

Widen, S. C., \& Russell, J. A. (2013). Children's recognition of disgust in others. Psychological bulletin, $139,271$. 